Acta Cryst. (1979). B35, 867-877

\title{
A Study of the Charge Density in Putrescine Diphosphate at $85 \mathrm{~K}^{*}$
}

\author{
By Fusao Takusagawa and Thomas F. Koetzle $\dagger$ \\ Chemistry Department, Brookhaven National Laboratory, Upton, New York 11973, USA
}

(Received 15 March 1978; accepted 7 September 1978)

\begin{abstract}
A study of the charge density in putrescine diphosphate $\left\{\mathrm{C}_{4} \mathrm{H}_{14} \mathrm{~N}_{2}^{2+} .2 \mathrm{H}_{2} \mathrm{PO}_{4}^{-},\left[\mathrm{NH}_{3}\left(\mathrm{CH}_{2}\right)_{4} \mathrm{NH}_{3}\right]^{2+} .2\left[\mathrm{H}_{2}-\right.\right.$ $\left.\left.\mathrm{PO}_{4}\right]^{-}\right\}$has been carried out, employing $\mathrm{X}$-ray and neutron diffraction data measured at a temperature of $85 \mathrm{~K}$. Crystal data: space group $P 2{ }_{1} / a ; a=7.879$ (3), $b=9.734$ (4), $c=8.126$ (3) A, $\beta=110.19$ (9) ${ }^{\circ}$ (Xray values); $Z=2$. The deformation electron density was computed by a modified $X-N$ method, utilizing neutron positional parameters for all atoms and thermal parameters for non-hydrogen atoms taken from a high-order $\mathrm{X}$-ray refinement $(0.65 \leq \sin \theta / \lambda \leq$ $1.15 \AA^{-1}$ ). This approach was elected, since the neutron thermal parameters were found to be systematically larger than those from high-order X-ray refinements. In the case of $\mathrm{H}$ atoms, the neutron thermal parameters were multiplied by the mean ratio of the X-ray and neutron values, computed separately for each $U_{i j}$ class for the non-hydrogen atoms. These ratios for the high-order $\mathrm{X}$-ray refinements range from 0.86 to 0.99 . Well-defined peaks $\left(0.25-0.35\right.$ e $\left.\AA^{-3}\right)$ are found in the deformation density maps for all covalent bonds in the structure. No marked difference was observed between the density in $\mathrm{P}-\mathrm{O}$ bonds and that in bonds between first-row elements. In the hydrogen bonds, no build up of charge was observed midway between the protons and oxygen acceptors, in accord with the basic electrostatic picture of hydrogen-bonded interactions. Excess density was observed in the regions normally associated with lone-pair electrons for all four phosphate $\mathrm{O}$ atoms, and the heights of these peaks increased more than those of peaks in covalent bonds when higher-order data were included in the deformation maps. Conformations of $\mathrm{H}_{2} \mathrm{PO}_{4}^{-}$ions in a number of crystal structures including putrescine diphosphate were generally found to have $\mathrm{H}-\mathrm{O}-$ $\mathrm{P}-\mathrm{O}(\mathrm{H})$ torsion angles in the range $30-150^{\circ}$; such conformations are predicted to be favored for systems like $\mathrm{H}_{2} \mathrm{PO}_{4}^{-}$with lone-pair electrons adjacent to polar bonds.
\end{abstract}

\footnotetext{
* Research carried out at Brookhaven National Laboratory under contract with the US Department of Energy and supported by its Office of Basic Energy Sciences.

+ To whom correspondence should be addressed.
}

\section{Introduction}

Recent improvements of the precision attainable in single-crystal diffraction measurements have led to increased interest in the direct analysis of chargedensity distributions in solids based on diffraction techniques (Coppens, 1975). Here we report a study of the electron density in putrescine diphosphate at $85 \mathrm{~K}$, using combined $\mathrm{X}$-ray and neutron diffraction data. This compound initially attracted our attention, because it affords the opportunity to study the charge density in the dihydrogen phosphate anion, and exhibits a variety of $\mathrm{N}-\mathrm{H} \cdots \mathrm{O}$ and $\mathrm{O}-\mathrm{H} \ldots \mathrm{O}$ hydrogen bonds involving the ammonium groups on putrescine and the phosphate $\mathrm{O}$ atoms. The crystal structure was originally determined from X-ray photographic data at room temperature (Woo \& Rich, 1975; Woo, Seeman $\&$ Rich, 1979). These authors have described the general features of the structure, and commented on its significance as a model for understanding interactions of amines with nucleic acids. Our neutron diffraction results have been described in a previous paper (Takusagawa \& Koetzle, 1978).

\section{Experimental}

The sample of putrescine diphosphate used in the present X-ray work was recrystallized from an aqueous solution of putrescine and phosphoric acid. A crystal was ground to form a sphere of diameter $0.42 \mathrm{~mm}$ which was glued to an aluminum fiber with adhesive varnish $\ddagger$ and oriented approximately along the crystallographic $c$ axis. This sample was placed in a liquid-nitrogen-cooled cryostat (Coppens et al., 1974) and mounted on an automated Picker four-circle diffractometer (Dimmler, Greenlaw, Kelley, Potter, Rankowitz \& Stubblefield, 1976; McMullan, Andrews, Koetzle, Reidinger, Thomas \& Williams, 1976). Nbfiltered Mo $K \alpha$ radiation $(\lambda=0.71069 \AA)$ was used. The temperature of a copper block in the cryostat, maintained in direct contact with the aluminum crystal mount, was measured to be $85 \pm 1 \mathrm{~K}$ during the period

$$
\mp \text { General Electric No. } 7031 \text {. }
$$


of data collection. Cell dimensions refined by a leastsquares procedure based on the setting angles of 29 reflections $\left(2 \theta \geq 80^{\circ}\right)\left(\right.$ Mo $\left.K \alpha_{1}: \lambda=0.70926 \AA\right)$ are listed along with certain experimental details and are compared with the corresponding neutron values in Table 1. X-ray intensities were measured for reflections in four octants of reciprocal space $(h k l, \bar{h} k l, h \bar{k} l$, and $\bar{h} \bar{k} l$ ), out to a $2 \theta$ limit of $110^{\circ}$. Mechanical constraints introduced by the cryostat prevented the measurement of reflections with $\chi$ near $90^{\circ}$ and $2 \theta>70^{\circ}$, but the number of reflections omitted was a small fraction of the total data measured. A $\theta / 2 \theta$ step-scan procedure was used; the scan range varied from 3.0 to $3.5^{\circ}$ over the full range of $2 \theta$, while the step size was taken to be $\Delta 2 \theta=0.04^{\circ}$. At each step, counts were accumulated for 2 s. As a general check on experimental stability, the intensities of two reflections were remeasured every 30 reflections. These intensities did not vary to any significant degree during the course of the measurements.

Integrated intensities were obtained with the program $P E A K_{1}$ (Takusagawa, 1977) written for a PDP $11 / 40$ computer equipped with an interactive CRT display (Bernstein et al., 1974; Vector General, Inc., 1973). A profile analysis was carried out for strong reflections with $I>15 \sigma(I)$, and the resulting peak widths were fitted by a least-squares procedure with a function of the type: $\Delta \theta=A x+B, x=\left[2\left(\lambda_{2}-\lambda_{1}\right) /\left(\lambda_{1}+\lambda_{2}\right)\right] \tan \theta$, where $\lambda_{1}(0.7093 \AA)$ and $\lambda_{2}(0.7136 \AA)$ are the wavelengths of Mo $\bar{K} \alpha_{1}$ and Mo $K \alpha_{2}$, respectively. $A$ and $B$ were determined to be 1.99 and $1.06^{\circ}$, respectively, based on approximately 12000 strong reflections. Calculated values of $\Delta \theta$ range from 1.06 to $1.45^{\circ}$ for 0 $<2 \theta<110^{\circ}$. The peak-width function defined above was used to determine trial points for the separation of peak and background regions in each individual scan. Final delineation of backgrounds was accomplished by the method of Lehmann \& Larsen (1974). At various points in the integration process, it was possible to

Table 1. Crystal data for putrescine diphosphate $(T=85 \mathrm{~K})$

$\mathrm{C}_{4} \mathrm{H}_{14} \mathrm{~N}_{2}^{2+} \cdot 2 \mathrm{H}_{2} \mathrm{PO}_{4}^{-}, \mathrm{FW} 284 \cdot 14$

\begin{tabular}{lll} 
& \multicolumn{1}{c}{ X-ray } & \multicolumn{1}{c}{ Neutron } \\
$a$ & $7.879(3) \AA$ & $7.890(7) \AA$ \\
$b$ & $9.734(4)$ & $9.725(8)$ \\
$c$ & $8.126(3)$ & $8 \cdot 132(8)$ \\
$\beta$ & $110 \cdot 19(9)^{\circ}$ & $110 \cdot 26(5)^{\circ}$ \\
$V$ & $584.9 \AA^{3}$ & $585.4 \AA^{3}$ \\
Space group & $P 21 / a$ & $P 2, a$ \\
$\rho_{c}$ & $1.614 \mathrm{Mg} \mathrm{m}^{-3}$ & $1.613 \mathrm{Mg} \mathrm{m}^{-3}$ \\
Crystal volume & $0.039 \mathrm{~mm}^{3}$ & $7.75 \mathrm{~mm}^{3}$ \\
(sin $\theta / \lambda)_{\max }$ & $1.153 \AA^{-1}$ & $0.675 \AA^{-1}$ \\
Total number of reflections & 16767 & 3208 \\
Unique reflections & 6840 & 1691 \\
Absorption coefficient & $40.85 \mathrm{~mm}^{-1}$ & $23.48 \mathrm{~mm}^{-1}$ \\
Transmission range & $0.840 \sim 0.864$ & $0.801 \sim 0.870$
\end{tabular}

examine selected profiles on the CRT, and certain reflections with unusual profiles were integrated manually.

Intensity data for several reflections collected as a function of rotation about the scattering vector indicated $2-3 \%$ variation in absorption. Therefore, observed intensities were corrected for absorption by a semi-empirical method (North, Phillips \& Mathews, 1968), based upon these $\psi$-scan measurements. Observed squared structure factors were calculated as $F_{0}^{2}=$ $2 I \sin 2 \theta /\left(1+\cos ^{2} 2 \theta\right)$ and averaged for symmetryrelated reflections. The agreement factor obtained is

$$
\begin{gathered}
R_{c}=\sum_{h k l}\left\langle\Delta F_{o}^{2}\right\rangle / \sum_{h k l}\left\langle F_{o}^{2}\right\rangle=0.022 \\
\left\langle\Delta F_{o}^{2}\right\rangle=\sum_{i=1}^{n}\left|\left\langle F_{o}^{2}\right\rangle-F_{o i}^{2}\right| / n
\end{gathered}
$$

where $n$ is the number of observations for a given reflection $h k l$ and its symmetry equivalents. The standard deviation of each reflection was estimated as follows: $\sigma^{2}\left(F_{0}^{2}\right)=\sigma^{2}$ (count) $+\left(A F_{0}^{2}\right)^{2}+\left(B F_{0}^{4}\right)^{2}$. Constants $A$ and $B$ were determined from $\left\langle\Delta F_{o}^{2}\right\rangle$ by a least-squares procedure minimizing the quantity $\sum_{h k l}\left[\left\langle\Delta F_{o}^{2}\right\rangle-\sigma^{2}\left(F_{0}^{2}\right)\right]^{2}$. The resulting values are $A=$ $1.34 \times 10^{-2}$ and $B=2.46 \times 10^{-8}$.

\section{$X$-ray refinements}

Initial positional parameters for all atoms were fixed at values determined in the neutron diffraction study (Takusagawa \& Koetzle, 1978). X-ray scattering factors for $\mathrm{P}, \mathrm{O}, \mathrm{N}$ and $\mathrm{C}$ atoms were taken from the relativistic Hartree-Fock values given in International Tables for X-ray Crystallography (1974); those for $\mathrm{H}$ atoms were from Stewart, Davidson \& Simpson (1965). For non-hydrogen atoms, the anomalousdispersion factors of Cromer \& Liberman (1970) were applied. A type I isotropic extinction correction (Becker \& Coppens, 1974) was included in the refinements. The minimum extinction correction factor is $y=$ 0.25 ( $y$ divides $F_{o}^{2}$ ) for the 001 reflection.

Conventional and high-order $\mathrm{X}$-ray refinements were carried out, as described in detail in Table 2. Procedures $1-5$ are 'pure' $X$-ray refinements, in which isotropic thermal parameters were assigned to $H$ atoms and anisotropic thermal parameters to non-hydrogen atoms. In procedures 6 and 7, positional parameters of all atoms and anisotropic thermal parameters of $\mathrm{H}$ atoms were fixed to the neutron values. The results of procedure 7 were used to calculate the deformation density, after suitable modification of the $\mathrm{H}$-atom thermal parameters, as described below. Atomic parameters obtained in the conventional $\mathbf{X}$-ray refine 
Table 2. Details of refinements

All calculations are full-matrix least-squares refinements, utilizing only reflections with $F_{a}^{2}>3 \sigma\left(F_{o}^{2}\right)$. The quantity minimized is $\Sigma w\left(F_{o}^{2}-\right.$ $\left.k^{2} F_{c}^{2}\right)^{2}$, where $w=1 / \sigma^{2}\left(F_{o}^{2}\right)$.

\begin{tabular}{|c|c|c|c|c|c|c|c|c|}
\hline Procedure & $\begin{array}{c}(\sin \theta / \lambda) \text { range } \\
\left(\AA^{-1}\right)\end{array}$ & $N_{o}$ & $N_{\nu}$ & $R\left(F^{2}\right)$ & $w R\left(F^{2}\right)$ & $S^{*}$ & Scale $(k)$ & $\begin{array}{l}\text { Maximum } \\
\text { correlation }\end{array}$ \\
\hline 1 & $0 \cdot 00-1 \cdot 15$ & 6258 & 110 & 0.027 & 0.025 & 1.91 & $15 \cdot 31(1)$ & 0.68 \\
\hline 2 & $0.65-1.15$ & 4975 & $73 \dagger$ & 0.033 & 0.029 & 1.81 & $15 \cdot 38(2)$ & 0.63 \\
\hline 3 & $0 \cdot 85-1 \cdot 15$ & 3463 & $72 \dagger$ & 0.043 & 0.037 & 1.83 & $15 \cdot 38 \S$ & 0.70 \\
\hline 4 & $1.00-1 \cdot 15$ & 1881 & $72 \dagger$ & 0.052 & 0.043 & 1.87 & $15 \cdot 38 \S$ & 0.62 \\
\hline 5 & $0.00-0.65$ & 1281 & 110 & 0.013 & 0.017 & $2 \cdot 10$ & $15 \cdot 22(4)$ & 0.79 \\
\hline 6 & $0.00-1 \cdot 15$ & 6258 & $50 \ddagger$ & 0.029 & 0.026 & 2.05 & $15 \cdot 33(1)$ & 0.68 \\
\hline 7 & $0.65-1.15$ & 4975 & $49 \ddagger 9$ & 0.035 & 0.031 & 1.89 & $15 \cdot 39(2)$ & 0.63 \\
\hline
\end{tabular}

* $S=\left[\Sigma w\left(F_{o}^{2}-k^{2} F_{c}^{2}\right)^{2} /\left(N_{o}-N_{\nu}\right)\right]^{1 / 2}$, where $N_{o}=$ number of observations, and $N_{v}=$ number of variables.

2.

$\ddagger$ All positional parameters and anisotropic thermal parameters for hydrogen atoms were fixed to the neutron results. Other parameters were refined.

$\S$ Not varied.

9 The extinction parameter was fixed to the results of refinement 6 .

Table 3. Fractional atomic coordinates and thermal parameters

$N=$ Neutron data. $X 1=\mathrm{X}$-ray data $\left(0.00 \leq \sin \theta / \lambda \leq 1 \cdot 15 \dot{\AA}^{-1}\right) . X 2=\mathrm{X}$-ray data $\left(0.65 \leq \sin \theta / \lambda \leq 1 \cdot 15 \dot{\AA}^{-1}\right) . X 3=\mathrm{X}$-ray data $\left(0.85 \leq \sin \theta / \lambda \leq 1.15 \AA^{-1}\right)$. The anisotropic Debye-Waller factor is of the form $\exp \left[-2 \pi^{2}\left(h^{2} a^{* 2} U_{11}+\cdots+2 h k a^{*} b^{*} U_{12}+\cdots\right)\right]$.

\begin{tabular}{|c|c|c|c|c|c|c|c|c|c|c|}
\hline & & $x$ & $y$ & $z$ & $U_{11}$ & $U_{22}$ & $U_{33}$ & $U_{12}$ & $U_{13}$ & $U_{23}$ \\
\hline \multirow[t]{4}{*}{$\mathbf{P}$} & $N$ & $0.84554(11)$ & $1.112562(68)$ & $0.120702(85)$ & $0.00625(34)$ & $0.00546(25)$ & $0.00752(27)$ & $-0.00010(23)$ & $0.00388(21)$ & $0.00055(21)$ \\
\hline & $x 1$ & 0.845608 (11) & $1.112566(8)$ & $0.120753(11)$ & $0.005604(23)$ & $0.005893(23)$ & $0.006304(28)$ & $-0.000122(17)$ & $0.003051(18)$ & $0.000391(18)$ \\
\hline & $x 2$ & $0.845613(12)$ & $1 \cdot 112558(9)$ & $0.120758(12)$ & $0.005677(25)$ & $0.005958(26)$ & $0.006398(31)$ & $-0.000111(18)$ & $0.003092(19)$ & $0.000396(18)$ \\
\hline & $x 3$ & $0.845613(16)$ & $1.112554(12)$ & $0.120760(17)$ & $0.005722(24)$ & $0.005967(24)$ & $0.006425(32)$ & $-0.000113(22)$ & $0.003107(21)$ & $0.000404(23)$ \\
\hline \multirow{4}{*}{$O(1)$} & $N$ & $0.66424(10)$ & 1. $118495(66)$ & $0.143343(84)$ & $0.00855(31)$ & $0.00893(24)$ & $0.01389(27)$ & 0.00103 (22) & $0.00696(20)$ & $0.00061(21)$ \\
\hline & $x 1$ & $0.663971(31)$ & $1 \cdot 118484(24)$ & $0.143367(34)$ & $0.007566(61)$ & $0.008792(65)$ & $0.012241(84)$ & $0.001070(50)$ & $0.006168(57)$ & $0.000716(55)$ \\
\hline & $x 2$ & $0.664019(34)$ & $1 \cdot 118506(27)$ & $0.143356(38)$ & $0.007619(62)$ & $0.008799(66)$ & $0.012252(87)$ & $0.001043(51)$ & $0.006144(58)$ & $0.000672(57)$ \\
\hline & $x^{3}$ & $0.664068(43)$ & $1.118508(35)$ & $0.143375(51)$ & $0.007685(70)$ & $0.008820(76)$ & $0.01238(11)$ & $0.001036(60)$ & $0.006198(69)$ & $0.000704(68)$ \\
\hline \multirow[t]{4}{*}{$O(2)$} & $N$ & 0.838771 (97) & $1.137247(64)$ & $-0.066036(78)$ & $0.00893(32)$ & $0.00843(24)$ & $0.00822(23)$ & $0.00027(21)$ & 0.00434 (19) & 0.00058 (19) \\
\hline & $x_{1}$ & $0.838693(31)$ & $1.137359(23)$ & $-0.066113(31)$ & $0.008231(61)$ & $0.008454(61)$ & $0.006522(71)$ & $0.000473(48)$ & $0.003541(51)$ & $0.000586(47)$ \\
\hline & $x 2$ & $0.838687(34)$ & $1.137344(25)$ & $-0.066098(34)$ & $0.008277(62)$ & $0.008557(62)$ & $0.006524(74)$ & $0.000445(48)$ & $0.003539(51)$ & $0.000554(48)$ \\
\hline & $x 3$ & $0.838709(43)$ & $1.137312(32)$ & $-0.066013(47)$ & $0.008331(71)$ & $0.008544(71)$ & $0.006703(92)$ & 0.000419 (56) & $0.003631(61)$ & $0.000533(57)$ \\
\hline \multirow{4}{*}{$O(3)$} & $N$ & 0.97695 (12) & $1.216356(81)$ & $0.248778(89)$ & $0.01788(38)$ & $0.01918(32)$ & $0.00987(25)$ & $-0.01107(27)$ & $0.00630(23)$ & $-0.00359(23)$ \\
\hline & $x 1$ & $0.976922(42)$ & i. $216363(32)$ & $0.249246(36)$ & $0.016547(98)$ & $0.01812(10)$ & $0.008416(87)$ & $-0.010753(81)$ & $0.005208(71)$ & $-0.003405(69)$ \\
\hline & $x 2$ & $0.976923(52)$ & $1.216374(40)$ & $0.249288(43)$ & $0.01653(10)$ & 0.01807 (11) & $0.008420(92)$ & $-0.010613(87)$ & $0.005172(75)$ & $-0.003347(72)$ \\
\hline & $x 3$ & $0.976962(73)$ & 1.216285 (57) & $0.249295(63)$ & $0.01661(13)$ & $0.01810(14)$ & $0.00845(12)$ & $-0.01053(11)$ & $0.005223(95)$ & $-0.003315(91)$ \\
\hline \multirow{4}{*}{$O(4)$} &.$N$ & 0.92057 (11) & $0.964394(71)$ & $0.182925(91)$ & $0.01470(37)$ & $0.01126(26)$ & $0.01687(29)$ & $0.00598(25)$ & $0.01067(23)$ & $0.00583(23)$ \\
\hline & $x 1$ & $0.920264(38)$ & $0.964186(27)$ & $0.182856(39)$ & $0.013908(84)$ & 0.010358 (73) & 0.015590 (97) & $0.005920(62)$ & $0.009957(74)$ & $0.005934(65)$ \\
\hline & $x 2$ & $0.920199(45)$ & $0.964177(31)$ & $0.182756(47)$ & $0.013916(88)$ & $0.010430(75)$ & 0.01554 (11) & $0.005873(65)$ & $0.009879(80)$ & $0.005887(69)$ \\
\hline & $x 3$ & $0.920105(61)$ & $0.964142(43)$ & $0.182566(67)$ & 0.01389 (ii) & $0.010465(90)$ & $0.01552(14)$ & $0.005813(81)$ & $0.00982(10)$ & $0.005871(87)$ \\
\hline \multirow[t]{4}{*}{$\mathrm{N}$} &.$N$ & $0.552751(61)$ & $0.868790(41)$ & $0.232165(49)$ & $0.00896(20)$ & $0.00818(16)$ & $0.00879(16)$ & $-0.00019(14)$ & $0.00403(12)$ & $-0.00086(12)$ \\
\hline & $x 1$ & $0.552758(35)$ & $0.868816(25)$ & $0.232226(35)$ & $0.008307(68)$ & $0.007953(68)$ & $0.006836(79)$ & -0.000071 (51) & $0.002998(56)$ & $-0.000694(51)$ \\
\hline & $x 2$ & $0.552796(37)$ & $0.868797(27)$ & $0.232218(38)$ & $0.008365(68)$ & $0.008043(69)$ & $0.006865(81)$ & $-0.000097(51)$ & 0.003007 (57) & $-0.000689(51)$ \\
\hline & $x 3$ & $0.552838(48)$ & $0.868800(34)$ & $0.232232(52)$ & $0.008436(78)$ & $0.008031(81)$ & $0.00707(10)$ & $-0.000074(59)$ & $0.003134(69)$ & $-0.000748(61)$ \\
\hline \multirow{4}{*}{$C(1)$} & $N$ & $0.634888(90)$ & $0.857174(59)$ & 0.426793 (71) & $0.01164(30)$ & $0.01037(22)$ & $0.00918(21)$ & $0.00291(20)$ & 0.00362 (18) & $0.00003(17)$ \\
\hline & $x_{1}$ & $0.634972(43)$ & $0.857295(33)$ & $0.426771(41)$ & 0.011145 (91) & $0.010084(85)$ & 0.007054 (96) & $0.002947(69)$ & $0.002631(72)$ & $0.000210(65)$ \\
\hline & $x 2$ & $0.634892(48)$ & $0.857290(36)$ & $0.426688(45)$ & $0.011187(92)$ & $0.010027(86)$ & $0.00730(10)$ & $0.002920(70)$ & $0.002727(73)$ & $0.000214(66)$ \\
\hline & $x 3$ & $0.634888(63)$ & $0.857266(48)$ & $0.426693(64)$ & $0.01129(11)$ & $0.01010(10)$ & $0.00756(13)$ & $0.002955(85)$ & $0.002852(91)$ & $0.000196(80)$ \\
\hline \multirow[t]{4}{*}{$C(2)$} & $N$ & $0.600127(88)$ & $0.985279(60)$. & $0.518474(69)$ & $0.01010(30)$ & 0.01144 (23) & $0.00934(23)$ & $0.00080(20)$ & $0.00328(18)$ & $-0.00213(18)$ \\
\hline & $x_{1}$ & 0.600205 (42) & $0.985181(32)$ & $0.518441(41)$ & $0.009065(82)$ & $0.010904(89)$ & 0.007777 (94) & $0.000759(67)$ & $0.002278(68)$ & $-0.002117(67)$ \\
\hline & $x 2$ & $0.600226(46)$ & $0.985189(35)$ & $0.518443(45)$ & $0.009086(83)$ & $0.010936(91)$ & $0.007819(98)$ & $0.000768(67)$ & $0.002225(69)$ & $-0.002062(67)$ \\
\hline & $x 3$ & $0.600208(59)$ & $0.985169(47)$ & $0.518438(63)$ & $0.009124(97)$ & 0.01093 (11) & $0.00786(13)$ & $0.000782(80)$ & $0.002218(84)$ & $-0.002014(82)$ \\
\hline \multirow[t]{2}{*}{$H(1)$} & $N$ & $0.59005(20)$ & $0.96205(13)$ & $0.18963(16)$ & $0.02459(73)$ & $0.01692(50)$ & $0.02193(53)$ & $-0.00257(46)$ & $0.01020(44)$ & $0.00265(41)$ \\
\hline & $x_{1}$ & 0.5903 (17) & 0.9521 (11) & $0.1936(16)$ & $0.0281(25)$ & & & & & \\
\hline \multirow[t]{2}{*}{$H(2)$} & $N$ & $0.41274(20)$ & $0.86459(15)$ & $0.18750(17)$ & $0.01491(65)$ & $0.02632(58)$ & $0.02204(54)$ & $-0.00044(50)$ & $0.00529(42)$ & $-0.00058(47)$ \\
\hline & $x_{1}$ & $0.4254(15)$ & $0.8622(11)$ & 0.1919 (15) & $0.0232(23)$ & & & & & \\
\hline \multirow{2}{*}{$H(3)$} & $N$ & $0.59604(22)$ & $0.79072(14)$ & $0.16892(17)$ & 0.02714 (77) & $0.01995(53)$ & $0.02128(54)$ & $0.00476(49)$ & $0.01112(46)$ & $-0.00512(43)$ \\
\hline & $x 1$ & 0.5900 (17) & 0.7988 (12) & 0.1725 (15) & $0.0277(25)$ & 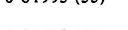 & & & & \\
\hline \multirow[t]{2}{*}{$H(4)$} & $N$ & $0.57892(25)$ & $0.76570(14)$ & $0.46777(19)$ & $0.04140(98)$ & $0.01780(54)$ & $0.02693(62)$ & $-0.00175(56)$ & $0.01649(57)$ & $0.00428(47)$ \\
\hline & $x_{1}$ & $0.5809(16)$ & $0.7723(11)$ & $0.4641(15)$ & $0.0215(23)$ & & & & & \\
\hline \multirow{2}{*}{$H(5)$} & $N$ & $0.78007(22)$ & $0.84265(18)$ & $0.45923(19)$ & $0.01715(73)$ & $0.03765(76)$ & $0.02626(61)$ & $0.00938(57)$ & $0.00368(47)$ & $-0.00316(54)$ \\
\hline & $x 1$ & $0.7714(14)$ & 0.8444 (11) & 0.4549 (14) & $0.0216(20)$ & & & & & \\
\hline \multirow{2}{*}{$H(6)$} & $N$ & $0.67068(23)$ & $0.96866(17)$ & $0.65932(17)$ & $0.02599(77)$ & $0.03700(72)$ & $0.01505(52)$ & $0.00728(61)$ & $0.00204(44)$ & $-0.00355(50)$ \\
\hline & $x 1$ & $0.6658(15)$ & 0.9674 (11) & $0.6504(16)$ & $0.0215(23)$ & & & & & \\
\hline$H(7)$ & $N$ & $0.66397(24)$ & $1.07422(15)$ & $0.48010(21)$ & $0.02660(86)$ & $0.02127(55)$ & $0.03565(72)$ & $-0.00585(53)$ & $0.01553(56)$ & $-0.00322(51)$ \\
\hline \multirow[b]{2}{*}{$H(8)$} & $x 1$ & 0.6629 (15) & $1.0619(13)$ & $0.4813(14)$ & $0.0294(23)$ & & & & & \\
\hline & $N$ & $1.05599(20)$ & $1.27515(14)$ & $0.20139(17)$ & $0.02066(69)$ & $0.02275(57)$ & $0.02276(54)$ & $-0.00614(49)$ & $0.00955(44)$ & $-0.00094(44)$ \\
\hline \multirow{3}{*}{$H(9)$} & $x 1$ & $1.0528(15)$ & 1.2728 (11) & 0.2035 (15) & $0.0243(24)$ & & & & & \\
\hline & $N$ & $1.01680(21)$ & $0.93124(14)$ & $0.13728(18)$ & $0.02112(74)$ & $0.02183(55)$ & $0.02648(59)$ & $0.00605(48)$ & $0.01321(47)$ & $0.00361(45)$ \\
\hline & $X 1$ & $1.0044(16)$ & $0.9373(13)$ & $0.1427(14)$ & $0.0288(25)$ & & & & & \\
\hline
\end{tabular}


ment (procedure 1), ${ }^{*}$ and in the refinements based on reflections with $\sin \theta / \lambda \geq 0.65 \AA^{-1}$ (procedure 2 ) and $\sin \theta / \lambda \geq 0.85 \AA^{-1}$ (procedure 3 ) are compared with the neutron values in Table 3 .

\section{Discussion of the structure}

The structure of putrescine diphosphate is shown in Fig. 1, which emphasizes the approximately tetrahedral arrangement of dihydrogen phosphate anions about the ammonium groups of putrescine. The packing exhibits three distinct $\mathrm{N}-\mathrm{H} \ldots \mathrm{O}$ and two $\mathrm{O}-\mathrm{H} \cdots \mathrm{O}$ hydrogen-bonded interactions; atom $\mathrm{O}(1)$ accepts two hydrogen bonds, while $\mathrm{O}(2)$ accepts three. This structure has been described in some detail in earlier papers (Woo et al., 1979; Takusagawa \& Koetzle, 1978). Here we make some comments comparing results of the $85 \mathrm{~K} \mathrm{X}$-ray and neutron refinements. Bond distances and angles calculated from the atomic coordinates obtained in the conventional Xray refinement are given in Table 4 along with the corresponding values from the neutron study. The

* A list of structure factors has been deposited with the British Library Lending Division as Supplementary Publication No. SUP 33945 (39 pp.). Copies may be obtained through The Executive Secretary, International Union of Crystallography, 5 Abbey Square, Chester CH1 2HU, England. agreement is in general quite good, except in the case of bond distances involving $\mathrm{H}$, which as expected are systematically short in the X-ray determination.

\section{Positional parameters}

The differences between the positions of nonhydrogen atoms found in the neutron study and those from X-ray refinements (1-5) are given in Table 5 (Xray asphericity shifts). The $\mathrm{C}, \mathrm{N}$ and $\mathrm{O}$ atoms show significant shifts in the low-order refinement (5),

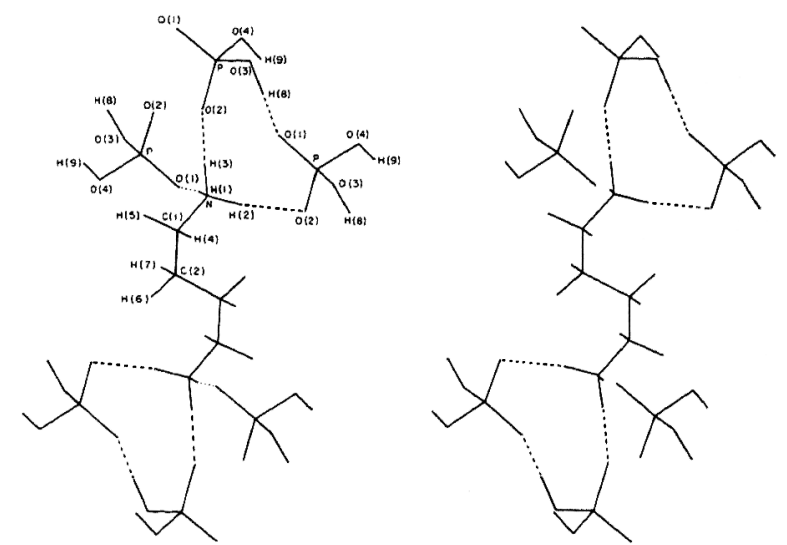

Fig. 1. Schematic stereoscopic representation of the crystal structure of putrescine diphosphate.

Table 4. Bond distances $(\AA)$ and angles $\left({ }^{\circ}\right)$

\begin{tabular}{|c|c|c|c|c|c|c|c|c|}
\hline & X-ray* & Neutron & & X-ray* & Neutron & & $\mathrm{X}$-ray* & Neutron \\
\hline $\mathrm{P}-\mathrm{O}(1)$ & $1.5056(6)$ & $1.5030(13)$ & $C(2)-C\left(2^{\prime}\right)$ & $1.5296(8)$ & $1 \cdot 5280(12)$ & $\mathrm{C}(1)-\mathrm{H}(5)$ & $1.03(1)$ & $1.091(2)$ \\
\hline$P-C(2)$ & $1.5198(6)$ & $1.5190(11)$ & $\mathrm{N}-\mathrm{H}(1)$ & $0.95(1)$ & $1.049(2)$ & $\mathrm{C}(2)-\mathrm{H}(6)$ & $1.03(1)$ & 1.098 (1) \\
\hline $\mathrm{P}-\mathrm{O}(3)$ & $1.5607(7)$ & $1.5595(12)$ & $\mathrm{N}-\mathrm{H}(2)$ & $0.94(1)$ & $1.036(2)$ & $\mathrm{C}(2)-\mathrm{H}(7)$ & $1.00(1)$ & 1.099 (2) \\
\hline $\mathrm{P}-\mathrm{O}(4)$ & $1.5754(6)$ & $1.5745(11)$ & $\mathrm{N}-\mathrm{H}(3)$ & $0.94(1)$ & $1.039(2)$ & $\mathrm{O}(3)-\mathrm{H}(8)$ & $0.97(1)$ & $1.015(2)$ \\
\hline $\mathrm{N}-\mathrm{C}(1)$ & $1.4915(9)$ & $1.4921(10)$ & $\mathrm{C}(1)-\mathrm{H}(4)$ & $1.02(1)$ & $1.095(2)$ & $\mathrm{O}(4)-\mathrm{H}(9)$ & $0.87(1)$ & $1.005(2)$ \\
\hline$C(1)-C(2)$ & $1.5233(7)$ & $1.5251(10)$ & & & & & & \\
\hline$O(1)-P-O(2)$ & $114.08(5)$ & $114 \cdot 14(7)$ & $\mathrm{C}(1)-\mathrm{N}-\mathrm{H}(2)$ & $112 \cdot 5(8)$ & $112 \cdot 8(1)$ & $\mathrm{C}(2)-\mathrm{C}(1)-\mathrm{H}(5)$ & $110 \cdot 0(6)$ & $109.8(1)$ \\
\hline $\mathrm{O}(1)-\mathrm{P}-\mathrm{O}(3)$ & $109.40(3)$ & $109.48(7)$ & $\mathrm{C}(1)-\mathrm{N}-\mathrm{H}(3)$ & $113.2(7)$ & $111.88(8)$ & $\mathrm{H}(4)-\mathrm{C}(1)-\mathrm{H}(5)$ & $110 \cdot 2(9)$ & 108.7 (1) \\
\hline $\mathrm{O}(1)-\mathrm{P}-\mathrm{O}(4)$ & $105 \cdot 16(2)$ & $105 \cdot 26(6)$ & $\mathrm{H}(1)-\mathrm{N}-\mathrm{H}(2)$ & $111(1)$ & $107 \cdot 7(1)$ & $\mathrm{C}(1)-\mathrm{C}(2)-\mathrm{H}(6)$ & $105 \cdot 1(6)$ & $106 \cdot 1(1)$ \\
\hline $\mathrm{O}(2)-\mathrm{P}-\mathrm{O}(3)$ & $110.64(4)$ & $110.52(7)$ & $\mathrm{H}(1)-\mathrm{N}-\mathrm{H}(3)$ & $105(1)$ & $107.0(1)$ & $\mathrm{C}(1)-\mathrm{C}(2)-\mathrm{H}(7)$ & $105.8(7)$ & $109.2(1)$ \\
\hline $\mathrm{O}(2)-\mathrm{P}-\mathrm{O}(4)$ & $110 \cdot 26(2)$ & $110 \cdot 22(6)$ & $\mathrm{H}(2)-\mathrm{N}-\mathrm{H}(3)$ & $105(1)$ & $106 \cdot 8(1)$ & $\mathrm{C}\left(2^{\prime}\right)-\mathrm{C}(2)-\mathrm{H}(6)$ & $110.4(8)$ & $110.5(1)$ \\
\hline $\mathrm{O}(3)-\mathrm{P}-\mathrm{O}(4)$ & $106.93(4)$ & $106.85(6)$ & $\mathrm{N}-\mathrm{C}(1)-\mathrm{H}(4)$ & $107 \cdot 6(6)$ & $108 \cdot 12(9)$ & $\mathrm{C}\left(2^{\prime}\right)-\mathrm{C}(2)-\mathrm{H}(7)$ & $112 \cdot 6(7)$ & $109.8(1)$ \\
\hline$N-C(1)-C(2)$ & $111.79(3)$ & $111.75(5)$ & $\mathrm{N}-\mathrm{C}(1)-\mathrm{H}(5)$ & $106 \cdot 5(6)$ & $107 \cdot 5(1)$ & $\mathrm{P}-\mathrm{O}(3)-\mathrm{H}(8)$ & $117 \cdot 5(7)$ & $117 \cdot 7(1)$ \\
\hline$C(1)-C(2)-C\left(2^{\prime}\right)$ & $113.95(4)$ & $113.87(5)$ & $\mathrm{C}(2)-\mathrm{C}(1)-\mathrm{H}(4)$ & $110.6(7)$ & $110.9(1)$ & $\mathrm{P}-\mathrm{O}(4)-\mathrm{H}(9)$ & $113.7(8)$ & 115.0 (1) \\
\hline $\mathrm{C}(1)-\mathrm{N}-\mathrm{H}(1)$ & $110 \cdot 1(7)$ & $110.28(8)$ & & & & & & \\
\hline
\end{tabular}

Table 5. X-ray asphericity shifts $\left(10^{-4} \AA\right)$

$\begin{array}{ccccccccrr}\text { Procedure } & \begin{array}{c}\sin \theta / \lambda) \text { range } \\ \left(\AA^{-1}\right)\end{array} & \mathrm{P} & \mathrm{O}(1) & \mathrm{O}(2) & \mathrm{O}(3) & \mathrm{O}(4) & \mathrm{N} & \mathrm{C}(1) & \mathrm{C}(2) \\ 1 & 0 \cdot 00-1 \cdot 15 & 6(4) & 22(9) & 13(7) & 39(9) & 30(9) & 5(5) & 14(7) & 12(7) \\ 2 & 0 \cdot 65-1 \cdot 15 & 6(4) & 18(9) & 12(7) & 42(9) & 35(9) & 5(4) & 14(7) & 13(7) \\ 3 & 0 \cdot 85-1 \cdot 15 & 6(4) & 15(9) & 9(8) & 44(10) & 44(8) & 6(5) & 12(8) & 14(8) \\ 4 & 1 \cdot 00-1 \cdot 15 & 5(5) & 20(9) & 16(9) & 56(13) & 62(9) & 5(9) & 8(11) & 12(11) \\ 5 & 0.00-0.65 & 5(4) & 34(10) & 29(8) & 43(10) & 43(10) & 22(9) & 20(11) & 16(10)\end{array}$


reflecting the contribution of valence electrons. By contrast, the P-atom shift is negligible, as expected due to its tetrahedral environment and the dominant influence of core electrons for a second-row element. In the high-order refinements $(2,3,4)$, shifts are reduced to the $2 \sigma$ level or below for all non-hydrogen atoms, with the exception of $\mathrm{O}(3)$ and $\mathrm{O}(4)$, the two protonated $\mathrm{O}$ atoms in the $\mathrm{H}_{2} \mathrm{PO}_{4}^{-}$ion. These latter shifts are of magnitude $0.004-0.006 \AA$, and lie in the direction of the expected lone-pair region on each $\mathrm{O}$ atom, thus causing relatively minor changes in $\mathrm{P}-\mathrm{O}$ distances (Table 6). Evidently, asphericity of the valence shell of $\mathrm{O}(3)$ and $\mathrm{O}(4)$ contributes significantly to reflections beyond $(\sin \theta / \lambda)=0.65 \AA^{-1}$. Theoretical evidence for such an effect has been given by Dawson (1964) and Coppens (1969), and the effect has been observed experimentally in sulfamic acid (Bats, Coppens \& Koetzle, 1977). It is, however, a little surprising that in the present work the asphericity shifts of $\mathrm{O}(3)$ and $\mathrm{O}(4)$ are observed to increase marginally upon raising the $(\sin \theta / \lambda)$ cut-off from $0.65 \AA^{-1}$ (procedure 2) to 1.00 $\AA^{-1}$ (procedure 4), rather than decreasing as one might expect, while $O(1)$ and $O(2)$ show no significant shifts, except in the low-order refinement (5).

\section{Thermal parameters}

Thermal parameters from the present $\mathrm{X}$-ray refinements generally are systematically smaller than those found in the neutron study (Takusagawa \& Koetzle, 1978).* The ratios of $\mathrm{X}$-ray and neutron $U_{i j}$ 's determined by least squares for the various $\mathrm{X}$-ray refinements are given in Table 7. There is little difference among the ratios determined for conventional and highorder refinements; depending upon the $U_{i j}$ class, X-ray parameters average from $1-14 \%$ lower than the corresponding neutron values. Systematic discrepancies between X-ray and neutron thermal parameters have been observed in a number of other crystals, e.g. ammonium tetraoxalate (Stevens, 1973; Currie, Speakman \& Curry, 1967), barbital II (Fox, Craven \& McMullan, 1977), $\alpha$-glycylglycine (Griffin \& Coppens, 1975; Kvick, Koetzle \& Stevens, 1979), parabanic acid (Fox, Craven \& McMullan, 1977), sodium azide (Stevens \& Hope, 1977; Choi \& Prince, 1976), and sulfamic acid (Bats et al., 1977). In ammonium tetra-

* See previous footnote. oxalate, $\alpha$-glycylglycine, sodium azide and sulfamic acid, some thermal parameters in the high-order X-ray refinements were always higher than the corresponding neutron values, contrary to the situation in parabanic acid, barbital II and in the present work on putrescine diphosphate, where the $\mathrm{X}$-ray thermal parameters were found to be lower than the neutron parameters. The origin of these differences is at present not well understood. They could in some cases partly be due to failure to adequately match the temperatures of the $\mathrm{X}$-ray and neutron experiments, but this is considered unlikely in the present work, since the samples used in both experiments were mounted directly on metal pins. At any rate, a temperature difference would produce a constant ratio for all $U_{i j}$ values (Coppens \& Vos, 1971) whereas the ratios in Table 7 show significant variation depending upon $U_{i j}$ class. It is interesting to note that the ratio departing furthest from unity is $U_{33}$, and that the crystal was mounted along the $c$ axis in the $\mathrm{X}$-ray experiment, and along [ $10 \overline{1}]$ in the neutron experiment. One probable source of error in the thermal parameters stems from thermal diffuse scattering (TDS), which has been shown to exert significant influence on refined thermal parameters, for the X-ray case (Helmholdt \& Vos, 1977). Neglect of TDS corrections would be expected to cause the thermal parameters to be smaller than their true values, and TDS might affect the X-ray and neutron experiments to a different extent.

\section{The deformation density}

A deformation electron-density map calculated from the observed X-ray structure factors with positional

\section{Table 7. Ratios of $X$-ray and neutron thermal parameters

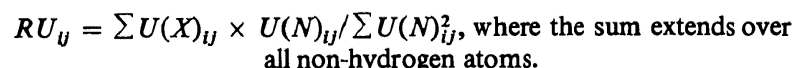

$\begin{array}{ccccccc}\text { Procedure } & R U_{11} & R U_{22} & R U_{33} & R U_{12} & R U_{13} & R U_{23} \\ 1 & 0.927 & 0.959 & 0.859 & 0.978 & 0.863 & 0.996 \\ 2 & 0.929 & 0.961 & 0.863 & 0.966 & 0.860 & 0.983 \\ 3 & 0.931 & 0.958 & 0.867 & 0.959 & 0.864 & 0.977 \\ 4 & 0.931 & 0.955 & 0.870 & 0.949 & 0.859 & 0.964 \\ 5 & 0.915 & 0.979 & 0.850 & 1.011 & 0.884 & 1.045 \\ 6 & 0.929 & 0.959 & 0.858 & 0.974 & 0.860 & 0.988 \\ 7 & 0.932 & 0.962 & 0.863 & 0.975 & 0.863 & 0.986\end{array}$

Table 6. $\mathrm{P}-\mathrm{O}$ bond distances $(\AA)$

\begin{tabular}{|c|c|c|c|c|c|c|}
\hline \multirow[b]{2}{*}{ Bond } & \multicolumn{4}{|c|}{$\mathrm{X}$-ray refinements } & \multirow[b]{2}{*}{5} & \multirow[b]{2}{*}{ Neutron } \\
\hline & 1 & 2 & 3 & 4 & & \\
\hline$P-O(1)$ & $1 \cdot 5056(6)$ & $1.5052(6)$ & $1.5049(7)$ & $1 \cdot 5050(8)$ & $1.5064(9)$ & $\begin{array}{l}1.5030(13) \\
1.5190(11)\end{array}$ \\
\hline $\mathrm{P}-\mathrm{O}(2)$ & $1.5198(6)$ & $1.5197(6)$ & $1.5190(7)$ & $1.5192(9)$ & $1.5212(8)$ & $1.5190(11)$ \\
\hline$P-O(3)$ & $1 \cdot 5607(7)$ & $1.5610(7)$ & $1.5607(8)$ & $1.5618(11)$ & $1.5611(9)$ & $1.5595(12)$ \\
\hline $\mathrm{P}-\mathrm{O}(4)$ & $1.5754(6)$ & $1.5751(6)$ & $1.5747(7)$ & $1.5753(9)$ & $1.5767(18)$ & $1.5745(11)$ \\
\hline
\end{tabular}


(i)
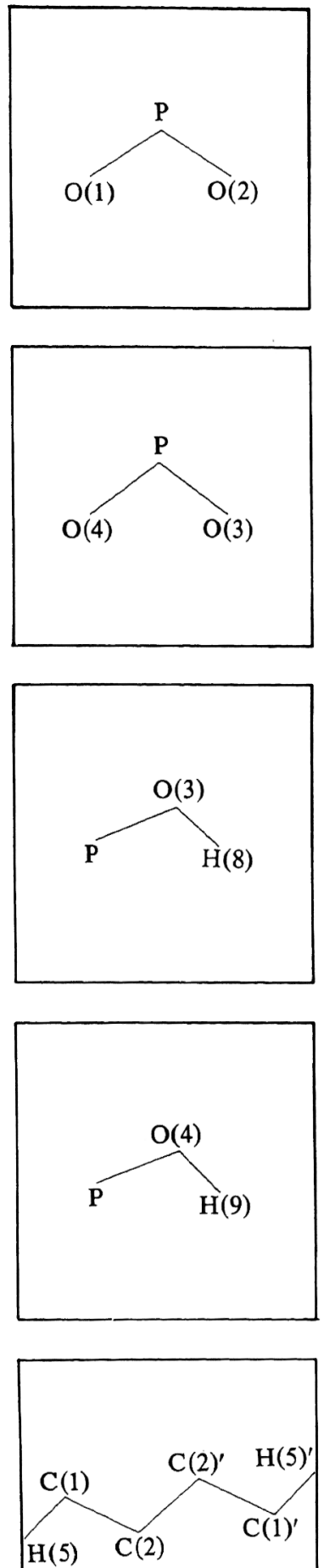

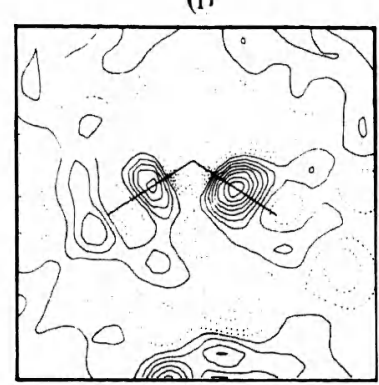

(a)

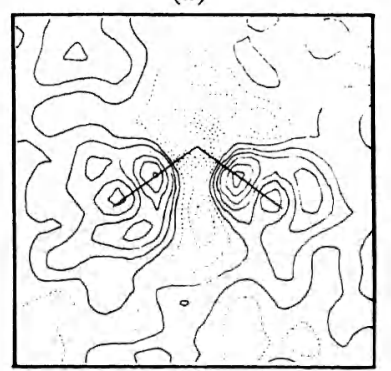

(b)

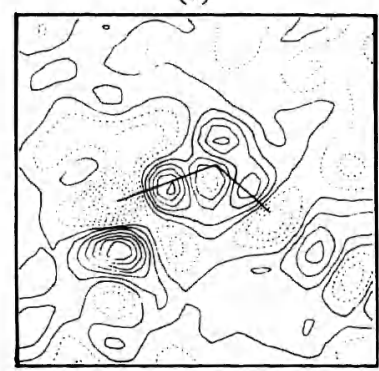

(c)

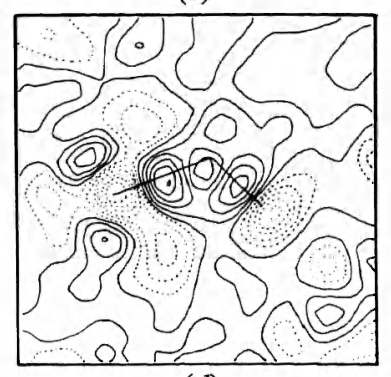

(d)

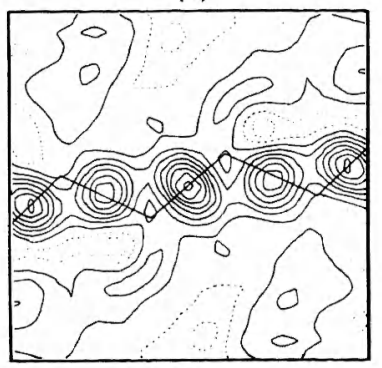

(e) (ii)

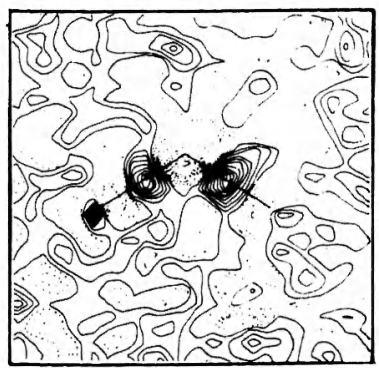

(a)

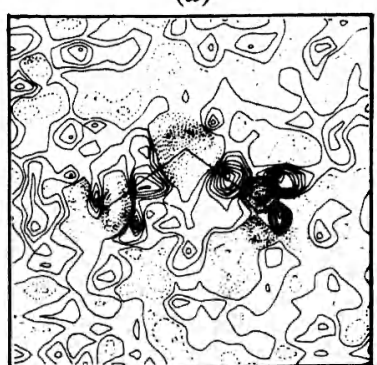

(b)

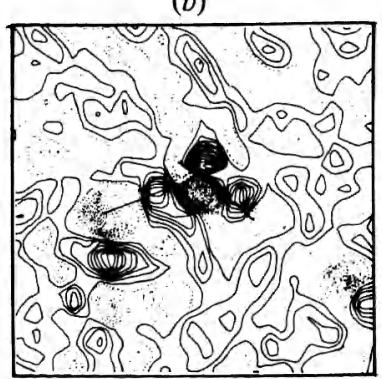

(c)

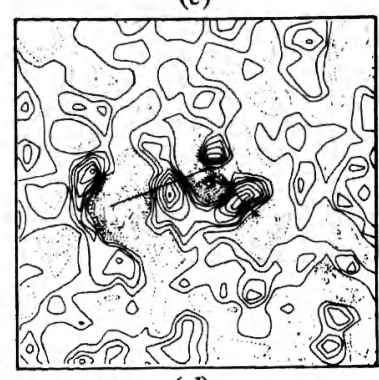

(d)

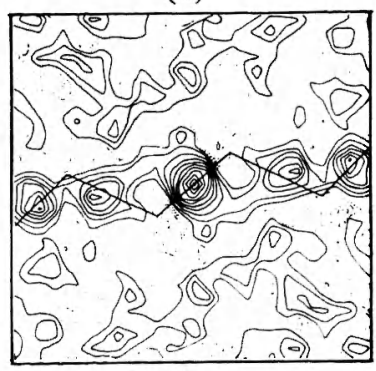

(e)

Fig. 2. Sections through the deformation density maps. (i) is calculated for the reflections with $\sin \theta / \lambda<0.65 \dot{A}^{-1}$, (ii) is calculated for th reflections with $\sin \theta / \lambda$ up to the experimental limit of $1.15 \AA^{-1}$. Contours are at intervals of $0.05 \mathrm{e} \dot{\mathrm{A}}^{-3}$, with negative contours dasted Positions of atoms used to define the section plane are indicated on the maps. 
(i)
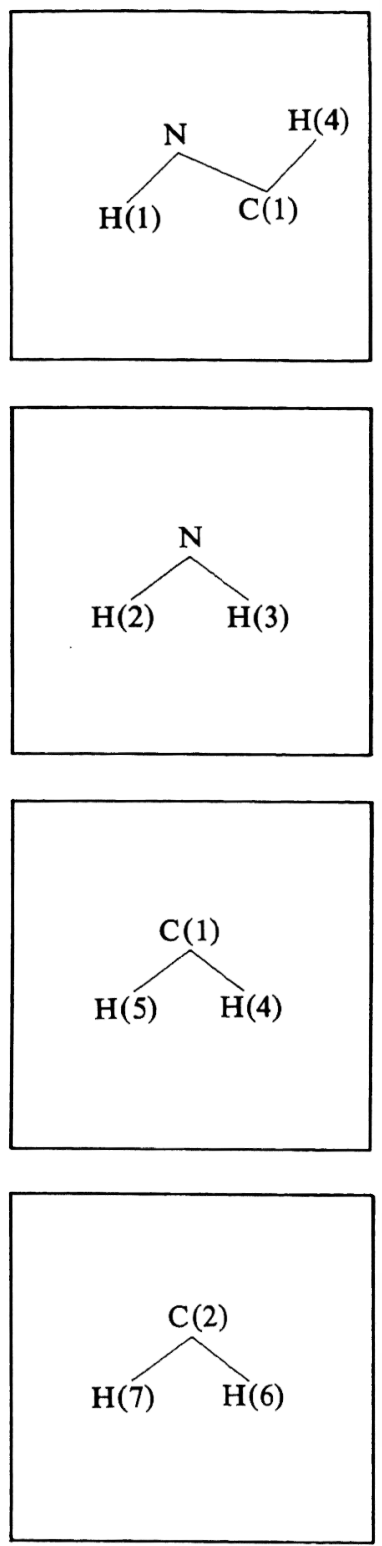

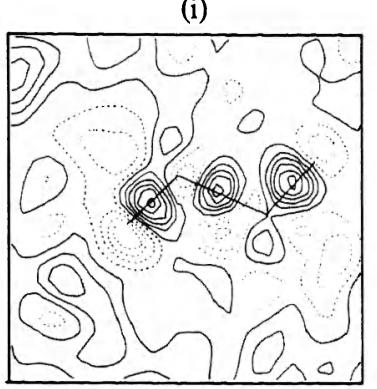

$(f)$

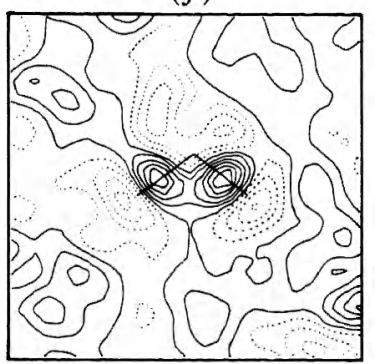

(g)

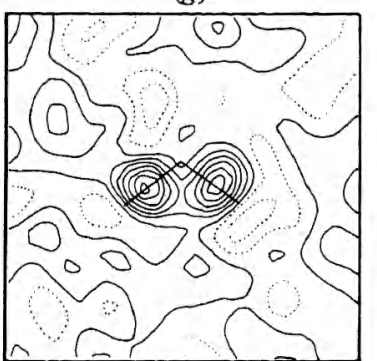

(h)

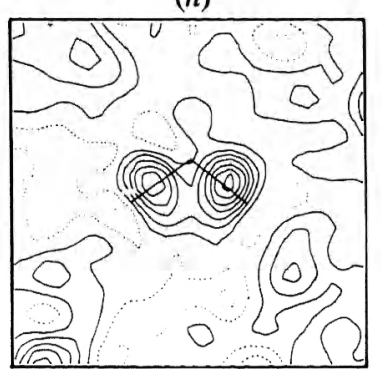

(i)

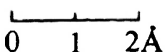

(ii)

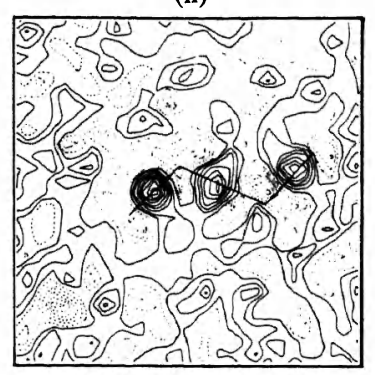

$(f)$

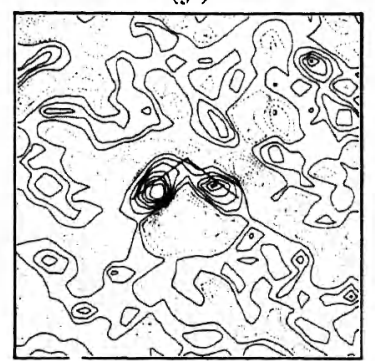

(g)

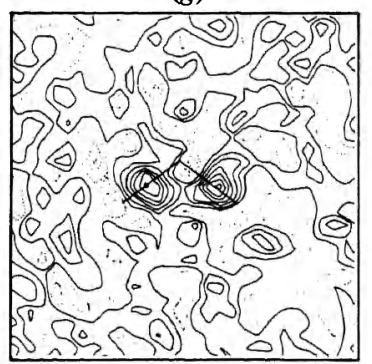

(h)

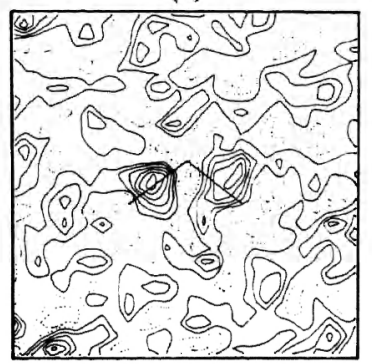

(i)

Fig. 2 (cont.)

and thermal parameters determined in the neutron study ( $X-N$ map) showed significant differences in features among chemically identical atoms and bonds. These differences are attributed to the systematic discrepancies between X-ray and neutron thermal parameters. Therefore, the results of procedure (7), a highorder X-ray refinement $\left[0.65 \leq(\sin \theta / \lambda) \leq 1.15 \AA^{-1}\right]$ in which thermal parameters of all non-hydrogen atoms have been varied together with the scale factor, were used to calculate modified $X-N$ deformation density maps. Anisotropic thermal parameters for $\mathrm{H}$ atoms were obtained by rescaling the neutron values with the appropriate ratio $R U_{i j}$ (Table 7), and the value of the $\mathrm{X}$-ray scale factor from refinement (7) was used to bring the observed structure factors onto an absolute scale. Maps were computed from difference structure 
factors $\Delta F_{X-N}=\left[F_{o}(X) / k\right]-F_{c}(N, \bmod ),{ }^{*}$ (i) for reflections with $(\sin \theta / \lambda) \leq 0.65 \AA^{-1}$ and (ii) for all reflections $\left[(\sin \theta / \lambda) \leq 1 \cdot 15 \AA^{-1}\right]$. A number of sections through the deformation density maps are displayed in Figs. 2-6. Inclusion of the high-order data in map (ii) (Figs. 2 and 4) appears to have increased the general level of noise in this map relative to that in map (i). Average variances were calculated by the equation (Coppens, 1974)

$$
\left\langle\sigma^{2}\left(\rho_{X-N}\right)\right\rangle=\frac{2}{V^{2}} \sum \sigma^{2}(|\Delta F|),
$$

yielding r.m.s. standard deviations $0.05 \mathrm{e} \AA^{-3}$ and 0.13 e $\AA^{-3}$ for maps (i) and (ii) respectively. Aside from the noise levels, the principal difference between the two maps is that peaks in lone-pair regions on $\mathrm{O}$ atoms, and to a lesser degree those between covalently bonded atoms, are observed to be higher in map (ii) than (i). Similar effects have been noted previously, for example in the case of $p$-nitropyridine $N$-oxide at $30 \mathrm{~K}$

* Reflections with $F_{e}^{2}\left(N\right.$,mod) $\left\langle 10 \sigma\left[F_{0}^{2}(X)\right]\right.$ and $\left.\Delta F^{2}(X-N)\right\rangle$ $3 \sigma\left[F^{2}(X)\right]$ were eliminated from the Fourier syntheses, as were those for which $F_{c}^{2}\left(N\right.$,mod) $>10 \sigma\left[F_{o}^{2}(X)\right]$ and $\Delta F^{2}(X-N)<$ $-20 \sigma\left[F_{o}^{2}(X)\right]$ or $\Delta F^{2}(X-N)>10 \sigma\left[F_{o}^{2}(X)\right]$. These criteria were intended to eliminate, respectively, weak reflections suspected of contamination by multiple scattering, and strong reflections with possibly inadequate extinction corrections or erroneous estimates of background. Altogether, 67 of a total of 1281 independent reflections were eliminated from map (i), and this resulted in signifcant improvement in the appearance of this map.

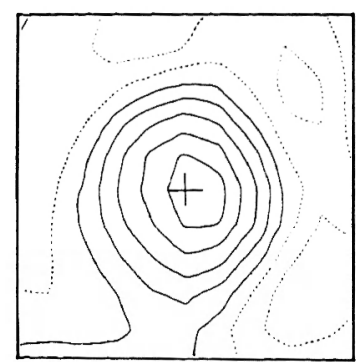

(a)

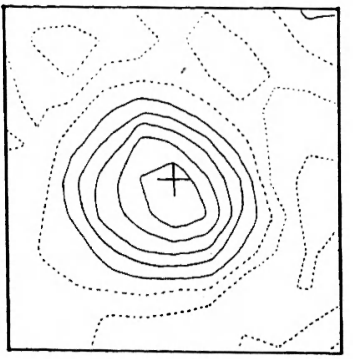

(c)

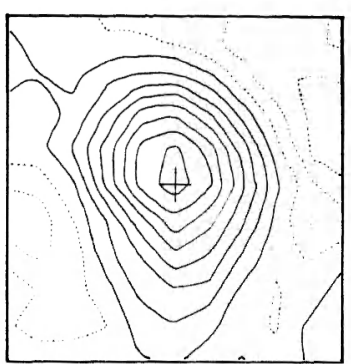

(b)

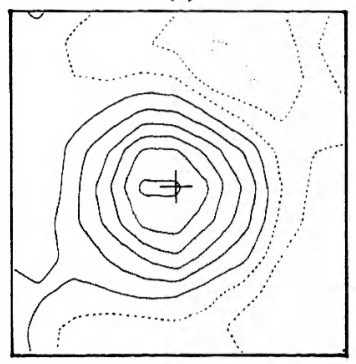

(d)

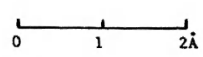

Fig. 3. Perpendicular sections through map (i) bisecting $P-O$ bonds, with projections of the bonds in question denoted by a cross, and contours as in Fig. 2. (a) $\mathrm{P}-\mathrm{O}(1),(b) \mathrm{P}-\mathrm{O}(2),(c)$ $\mathrm{P}-\mathrm{O}(3),(d) \mathrm{P}-\mathrm{O}(4)$. (i)

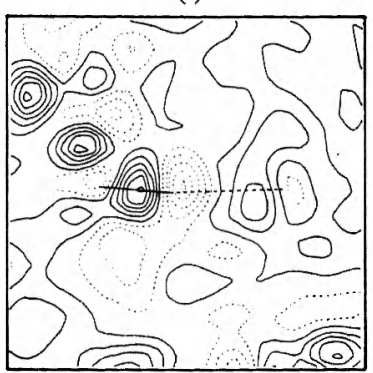

(a)

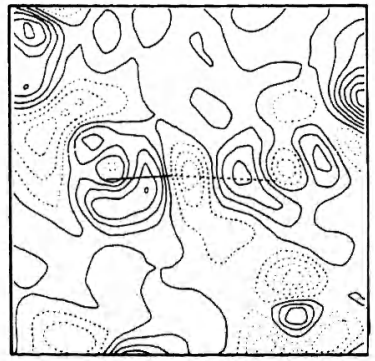

(b)

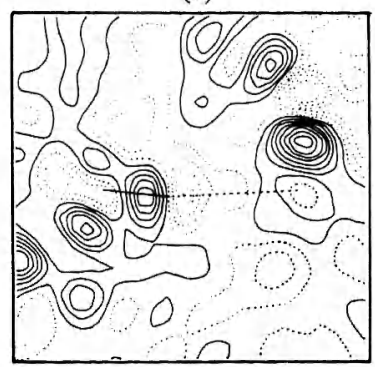

(c)

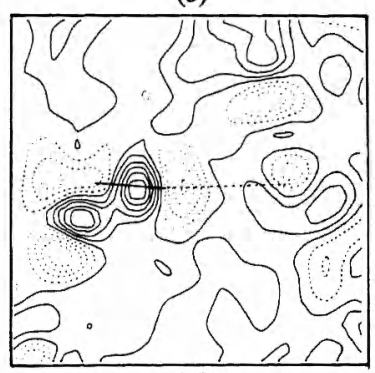

(d)

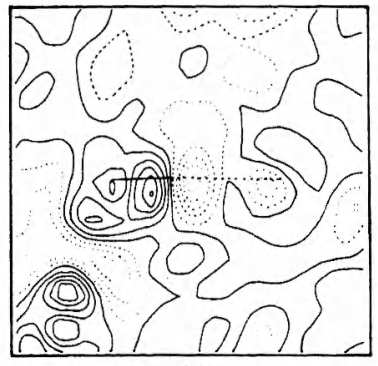

(e)

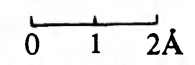

Fig. 4. Sections of maps (i) and (ii), taken through the hydrogen bonds in the structure, with contours as in Fig. 2. (a) $\mathrm{N}-\mathrm{H}(1) \cdots$ $\mathrm{O}(1), \quad$ (b) $\mathrm{O}(3)-\mathrm{H}(8) \cdots \mathrm{O}(1), \quad$ (c) $\mathrm{N}-\mathrm{H}(2) \cdots \mathrm{O}(2), \quad$ (d) $\mathrm{N}-\mathrm{H}(3) \cdots \mathrm{O}(2),(e) \mathrm{O}(4)-\mathrm{H}(9) \cdots \mathrm{O}(2)$. (ii)

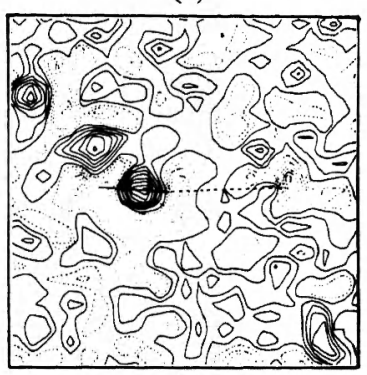

(a)

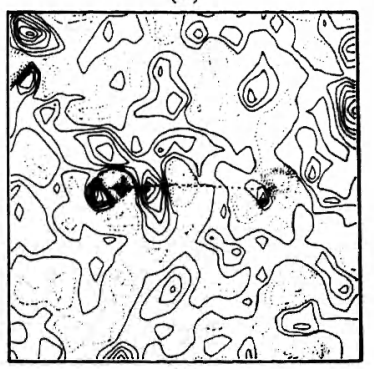

(b)

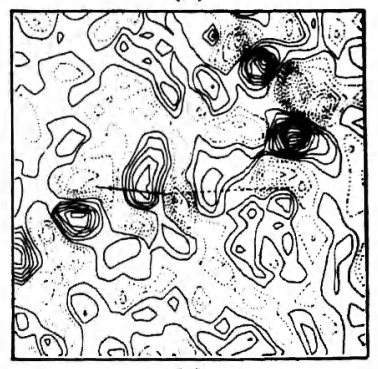

(c)

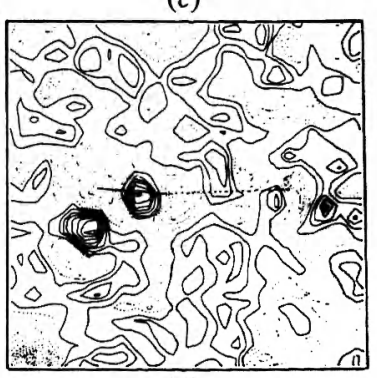

(d)

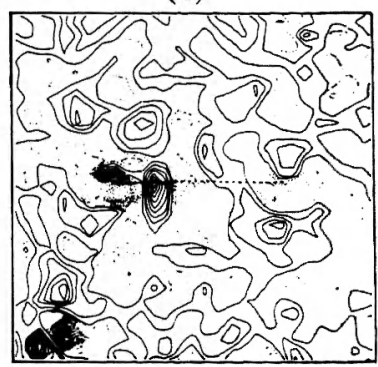

(e) 
(Coppens \& Lehmann, 1976). For putrescine diphosphate, the low-order deformation map (i) shows somewhat better reproducibility among chemically identical features than does the high-order map (ii). For this reason, the qualitative discussions which follow are based principally on the low-order map. It is however important to realize that the peaks in the low-order map are systematically reduced by series-termination effects. Well-defined peaks $\left(0.25\right.$ to $\left.0.35 \mathrm{e}^{-3}\right)$ are found in all covalent bonds in both the putrescine and dihydrogen phosphate ions (Fig. 2). Peak densities in the $\mathrm{C}-\mathrm{C}$, $\mathrm{C}-\mathrm{N}, \mathrm{C}-\mathrm{H}$ and $\mathrm{N}-\mathrm{H}$ bonds of putrescine are comparable to those found in other compounds (see, for example, Coppens, 1974). Cross-sections through the $\mathrm{P}-\mathrm{O}$ bonds are shown in Fig. 3, which indicates the approximately cylindrical symmetry of the density in these bonds. Peak densities are found to be: $\mathrm{P}-\mathrm{O}(1)$ $0.20, \mathrm{P}-\mathrm{O}(2) 0.35, \mathrm{P}-\mathrm{O}(3) 0.20$, and $\mathrm{P}-\mathrm{O}$ (4) $0.25 \mathrm{e}$ $\AA^{-3}$. While there can be little doubt that the overlap density associated with the $\mathrm{P}-\mathrm{O}$ bonds (mean distance $1.51 \AA$ A) should be larger than that associated with $\mathrm{P}-\mathrm{OH}(1.57 \AA)$, it is not possible to discriminate between the two types of bonds, based on peak heights in the present deformation density maps. However, it is important to note that differences in overlap density
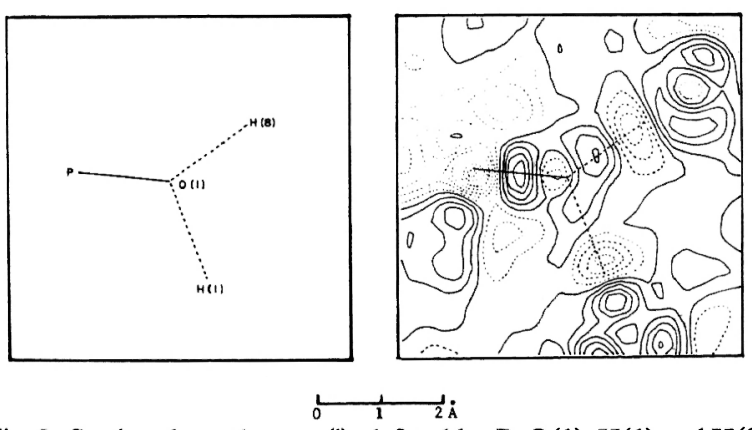

Fig. 5. Section through map (i), defined by $P, O(1), H(1)$ and $\mathrm{H}(8)$, with contours as in Fig. 2.

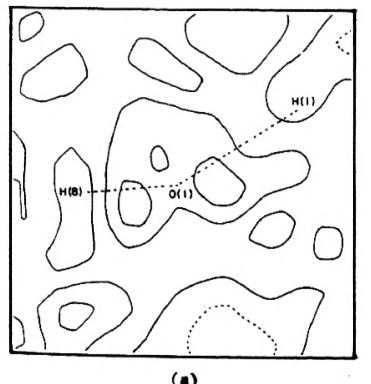

(a)

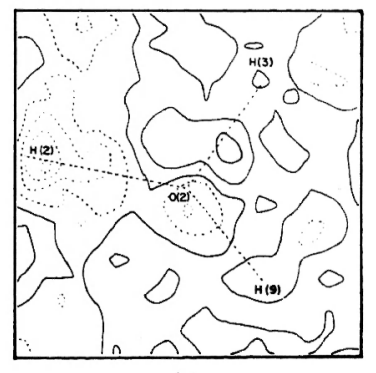

(b)

\section{$\underbrace{1}_{0}$}

Fig. 6. Sections through map (i), showing the lone-pair regions of (a) $\mathrm{O}(1)$ and $(b) \mathrm{O}(2)$. The section planes are normal to the $\mathrm{P}-\mathrm{O}$ bonds, displaced $0.1 \AA$ beyond the $\mathrm{O}$ atoms, contours as in Fig. may tend to be obscured in such deformation maps, since more density is subtracted at a bond midpoint if the corresponding atoms are closer together.

Sections of the deformation map, taken through the hydrogen bonds in the crystal are shown in Fig. 4. These sections show that there is no significant build up of density midway between the protons and $O$ acceptors, thus supporting an electrostatic model of hydrogen-bonded interactions. Similar observations have been made for several other hydrogen-bonded crystals [e.g. 2-amino-5-chloropyridine (Kvick, Thomas \& Koetzle, 1976) and (x-glycine (Almlöf, Kvick \& Thomas, 1973)].

It is interesting to examine the charge distribution in regions normally associated with $\mathrm{O}$-atom lone-pair density in the present structure. In the case of $O(1)$, there are two peaks at approximately $120^{\circ}$ angles, pointing in the general directions of the hydrogen bonds, as is shown in Figs. 5 and 6(a). These two peaks are of substantially the same height $\left[0.10-0.15\right.$ e $\AA^{-3}$ in map (i)], and presumably reflect the polarization of the $O$ valence shell by the crystal field. In contrast to the situation observed for $O(1)$, the density in the lonepair region on $\mathrm{O}(2)$ is not well-localized, but rather forms a ring of charge (see Fig. $6 b$ ). A peak $\left[0.10 \mathrm{e} \AA^{-3}\right.$ in map (i)] occurs in the direction of one of the three hydrogen bonds, that involving $\mathbf{H}(3)$. In this structure, there does not appear to be any systematic correlation between hydrogen-bond length and the amount of excess density near the $\mathrm{O}$ atom along the corresponding direction; existence of an inverse correlation has recently been suggested by Thomas (1977).

The lone-pair density associated with both hydroxyl $\mathrm{O}$ atoms, $\mathrm{O}(3)$ and $\mathrm{O}(4)$, is quite pronounced as shown in Fig. 2(c), (d). In particular, the large peaks ( $c a 1.0 \mathrm{e}$ $\AA^{-3}$ ) and corresponding holes in the deformation map (ii) including high-order data are related to the observed asphericity shifts for these atoms (Table 5).* Situations such as occur here, with lone-pair electrons adjacent to polar bonds, have been shown (Radom, Hehre \& Pople, 1972) to negate the normal preference for trans conformations. Recently, Hayes, Kollman \& Rothenberg (1977) have performed a conformational analysis of $\mathrm{H}_{2} \mathrm{PO}_{4}^{-}$by ab initio methods, and calculated that conformations with $\mathrm{H}-\mathrm{O}-\mathrm{P}-\mathrm{O}(\mathrm{H})$ torsion angles in the neighborhood between 60 and $120^{\circ}$ are favored by about $25 \mathrm{~kJ} \mathrm{~mol}^{-1}$ relative to conformations with both torsion angles equal to 0 or $180^{\circ}$. These results are somewhat similar to those obtained by Newton (1973) for $\left(\mathrm{CH}_{3}\right)_{2} \mathrm{PO}_{4}$, where the barrier was calculated to be approximately $29 \mathrm{~kJ} \mathrm{~mol}^{-1}$. In

\footnotetext{
* In order to check the possibility that these features might be due to anharmonic motion, refinements were carried out incorporating third-order thermal tensors (Johnson, 1969, 1970) for the $O$ atoms, but the resulting third-order terms did not differ from zero by more than one e.s.d. for either the X-ray or neutron calculations.
} 
Table 8. Observed conformational angles in $\mathrm{H}_{2} \mathrm{PO}_{4}^{-}$ ions

L-Arginine phosphate monohydrate ${ }^{a}$

(-)-Ephedrine dihydrogen phosphate ${ }^{b}$

Glycylglycine phosphate monohydrate ${ }^{c}$

Histamine diphosphate monohydrate $\dagger^{d}$

Imidazolium dihydrogen orthophosphate $\dagger^{e}$

Methylguanidinium dihydrogen orthophosphate

Putrescine diphosphate ${ }^{8}$

Procaine dihydrogen orthophosphate ${ }^{h}$

References: (a) Saenger \& Wagner (1972). (b) Hearn \& Bugg (1972). (c) Freeman, Hearn \& Bugg (1972). (d) Veidis, Palenik, Schaffrin \& Trotter (1969). (e) Blessing \& McGandy (1972). $(f)$ Cotton, Day, Hazen \& Larsen (1973). (g) Takusagawa \& Koetzle (1978). (h) Freeman \& Bugg (1975).

* $\varphi_{1}$ has arbitrarily been assigned as the larger of the two $\mathrm{H}-\mathrm{O}-\mathrm{P}-\mathrm{O}(\mathrm{H})$ torsion angles in each anion. Torsion angles were calculated according to the IUPAC-IUB Commission on Biochemical Nomenclature (1970) recommendations.

$\dagger$ Structure includes two independent $\mathrm{H}_{2} \mathrm{PO}_{4}^{-}$ions.

putrescine diphosphate, the observed conformation has $\varphi_{1}=\mathrm{H}(8)-\mathrm{O}(3)-\mathrm{P}-\mathrm{O}(4)=110^{\circ}, \varphi_{2}=\mathrm{H}(9)-\mathrm{O}(4)-$ $\mathrm{P}-\mathrm{O}(3)=-85^{\circ}$, and lies within $0.4 \mathrm{~kJ} \mathrm{~mol}^{-1}$ of the minimum energy calculated by Hayes, Kollman \& Rothenberg (1977) for $\varphi_{1}=\varphi_{2}=120^{\circ}$. An automated search of the Cambridge bibliographic data file for organic and organometallic compounds (Kennard, Watson \& Town, 1972) using the Chemical Information System (Heller, Milne \& Feldmann, 1977) has revealed seven additional structures with $\mathrm{H}_{2} \mathrm{PO}_{4}^{-}$ions, for which positions of $\mathrm{H}$ atoms are known from $\mathrm{X}$-ray diffraction data. Conformational angles for the ten $\mathrm{H}_{2} \mathrm{PO}_{4}^{-}$ions in these structures are listed in Table 8. Of these, eight $\mathrm{H}_{2} \mathrm{PO}_{4}^{-}$ions have conformations lying within $4 \mathrm{~kJ} \mathrm{~mol}^{-1}$ of the minimum calculated energy (Hayes et al., 1977) while conformations of two ions [those in (-)-ephedrine dihydrogen phosphate and glycylglycine phosphate monohydrate] lie in relatively unfavorable regions. Thus, in spite of the moderately strong hydrogen bonds and other intermolecular interactions present in these crystal structures, in general intramolecular electronic effects apparently play a dominant role in determining the conformations of the $\mathrm{H}_{2} \mathrm{PO}_{4}^{-}$ions.

We wish to acknowledge helpful discussions with Marshall Newton. We thank Joseph Henriques for technical assistance and Herbert Bernstein for carrying out the computer search of the Cambridge bibliographic file.

\section{References}

Almlöf, J., Kvick, А. \& Thомas, J. O. (1973). J. Chem. Phys. 59, 3901-3906.

Bats, J. W., Coppens, P. \& Koetzle, T. F. (1977). Acta Cryst. B33, 37-45.

Becker, P. J. \& Coppens, P. (1974). Acta Cryst. A31, 417425.

Bernstein, H. J., Andrews, L. C., Berman, H. M., Bernstein, F. C., Campbell, G. H., Carrell, H. L., Chiang, H. B., Hamilton, W. C., Jones, D. D., Klunk, D., Koetzle, T. F., Meyer, E. F., Morimoto, C. N., Sevian, S. S., Stodola, R. K., Strongson, M. M. \& Willoughby, T. V. (1974). Second Annual AEC Scientific Computer Information Exchange Meeting, Proceedings of the Technical Program, pp. 148-158. Report BNL 18803. Brookhaven National Laboratory, Upton, New York.

Blessing, R. H. \& McGandy, E. L. (1972). J. Am. Chem. Soc. 94, 4034-4035.

Choi, C. S. \& Prince, E. (1976). J. Chem. Phys. 64, 45104516.

Coppens, P. (1969). Acta Cryst. A25, 180-186.

Coppens, P. (1974). Acta Cryst. B30, 255-261.

Coppens, P. (1975). Measurement of Electron Densities in Solids by X-ray Diffraction. MTP Int. Rev. Sci. 11, Ser. 2, pp. 21-56.

Coppens, P. \& Lehmann, M. S. (1976). Acta Cryst. B32, 1777-1784.

Coppens, P., Ross, F. K., Blessing, R. H., CoOper, W. F., LARSEN, F. K., LeIPOLDT, J. G., ReES, B. \& LeONARD, R. (1974). J. Appl. Cryst. 7, 315-319.

Coppens, P. \& Vos, A. (1971). Acta Cryst. B27, 146158.

Cotton, F. A., Day, V. W., Hazen, E. E. JR \& Larsen, S. (1973). J. Am. Chem. Soc. 95, 4834-4840.

Cromer, D. T. \& Liberman, D. (1970). J. Chem. Phys. 53, 1891-1898.

Currie, M., Speakman, J. C. \& Curry, N. A. (1967). $J$. Chem. Soc. A, pp. 1862-1869.

Dawson, B. (1964). Acta Cryst. 17, 997-1009.

Dimmler, D. G., Greenlaw, N., Kelley, M. A., Potter, D. W., Rankowitz, S. \& Stubblefield, F. W. (1976). IEEE Trans. Nucl. Sci. NS23, 398-405.

Fox, R. O., Craven, B. M. \& McMullan, R. K. (1977). Abstracts, Am. Crystallogr. Assoc. Summer Meeting, East Lansing, Michigan, pp. 81-82.

Freeman, G. R. \& Bugg, C. E. (1975). Acta Cryst. B31, 96-101.

Freeman, G. R., Hearn, R. A. \& Bugg, C. E. (1972). Acta Cryst. B28, 2906-2912.

Griffin, J. F. \& Coppens, P. (1975). J. Am. Chem. Soc. 97 , 3496-3505.

Hayes, D. M., Kollman, P. A. \& Rothenberg, S. (1977). J. Am. Chem. Soc. 99, 2150-2154.

Hearn, R. A. \& BugG, C. E. (1972). Acta Cryst. B28, 3662-3667.

Heller, S. R., Milne, G. W. A. \& FeldmanN, R. J. (1977). Science, 195, 253-259.

Helmhold,, R. B. \& Vos, A. (1977). Acta Cryst. A33, 456-465.

International Tables for X-ray Crystallography (1974). Vol. IV, pp. 71-147. Birmingham: Kynoch Press. 
IUPAC-IUB COMMISSION ON BIOCHEMICAL NOMENClATURE (1970). J. Biol. Chem. 245, 6489-6497.

Johnson, C. K. (1969). Acta Cryst. A25, 187-194.

Johnson, C. K. (1970). Thermal Neutron Diffraction, edited by B. T. M. Willis, pp. 132-160. Oxford Univ. Press.

KennaRd, O., WATSON, D. G. \& Town, W. G. (1972). $J$. Chem. Doc. 12, 14-19.

Kvick, Á., Koetzle, T. F. \& Stevens, E. D. (1979). To be published.

Kvick, A., Thomas, R. \& Koetzle, T. F. (1976). Acta Cryst. B32, 224-231.

Lehmann, M. S. \& Larsen, F. K. (1974). Acta Cryst. A30, 580-584.

McMullan, R. K. AND, IN PART, Andrews, L. C., Koetzle, T. F., Reidinger, F., Thomas, R. \& Williams, G. J. B. (1976). Neutron and X-ray Data Acquisition System (NEXDAS). Unpublished work.

Newton, M. D. (1973). J. Am. Chem. Soc. 95, 256-258.

North, A. C. T., Phillips, D. C. \& Mathews, F. S. (1968). Acta Cryst. A24, 351-359.

Radom, L., Hehre, W. J. \& Pople, J. A. (1972). J. Am. Chem. Soc. 94, 2371-2381.
Saenger, W. \& W Agner, K. G. (1972). Acta Cryst. B28, 2237-2244.

Stevens, E. D. (1973). Thesis, Univ. of California.

Stevens, E. D. \& Hope, H. (1977). Acta Cryst. A33, 723729.

Stewart, R. F., Davidson, E. R. \& Simpson, W. T. (1965). J. Chem. Phys. 42, 3175-3187.

TAKUSAGAWA, F. (1977). Unpublished work.

Takusagawa, F. \& Koetzle, T. F. (1978). Acta Cryst. B34, 1910-1915.

Thomas, J. O. (1977). Acta Cryst. B33, 2867-2876.

Vector General, Inc. (1973). Fortran Drawing Package Reference Manual, 8399 Topanga Canyon Blvd., Canoga Park, California 91304.

Veidis, M. V., Palenik, G. J., Schaffrin, R. \& Trotter, J. (1969). J. Chem. Soc. A, pp. 2659-2666.

Woo, N. \& Rich, A. (1975). Abstracts, Am. Crystallogr. Assoc. Spring Meeting, Charlottesville, Virginia, p. 13.

Woo, N., SeEman, N. C. \& Rich, A. (1979). Biopolymers. In the press.

Acta Cryst. (1979). B35, 877-881

\title{
The Crystal Structure of $N, N^{\prime}$-Dibenzyl- $N$-diphenoxyphosphorylthiourea
}

\author{
BY JANINA KAROLAK-WOJCIECHOWSKA AND MICHAE W IECZOREK \\ Institute of General Chemistry, Technical University, 90-362 Eódż, Zwirki 36, Poland \\ Marian MikolajczyK ANd Piotr Kielbasiński \\ Centre of Molecular and Macromolecular Studies, Polish Academy of Sciences, \\ Department of Organic Sulphur Compounds, 90-362 Eódź, Boczna 5, Poland
}

AND YURIJ T. StruchKov AND M. Y. ANTIPIN

Institute of Organoelement Compounds, USSR Academy of Sciences, 28 Vavilov Street, Moscow 117312, USSR

(Received 30 August 1978; accepted 24 October 1978)

\begin{abstract}
Crystals of the title compound are triclinic, space group $P \overline{1}, a=8.0101(8), b=11.8192(9), c=13.5001$ (9) $\AA, \alpha=97.27(9), \beta=100.79(9), \gamma=101.16(9)^{\circ}, Z=$ 2. 2591 intensities were collected on a Syntex diffractometer. The structure was solved by the direct method. The positions of all the $\mathrm{H}$ atoms were shown by a Fourier synthesis. The final $R$ value was 0.033 . The positions of the $\mathrm{H}$ atoms were refined with isotropic temperature factors. The non-hydrogen atoms were refined anisotropically. The atoms forming the
\end{abstract}

0567-7408/79/040877-05\$01.00 thiourea system are coplanar. There is a hydrogen bond between the phosphoryl $\mathrm{O}$ atom and the secondary $\mathrm{N}$ atom of length $2.702 \AA$.

\section{Introduction}

In connexion with a research programme on the reaction between carbodiimides and thio- and seleno-acids (Mikolajczyk, Kielbasiński \& Schiebel, 1976; Mikolajczyk, Kielbasiński, Barlow \& Russell, 1977; Mikolajczyk, Kielbasiński \& Goszczyńska, 1977) we (C) 1979 International Union of Crystallography 